\title{
Combinatorial Analysis of mRNA Expression Patterns in Mouse Embryos Using Hybridization Chain Reaction
}

\author{
David Huss, ${ }^{1}$ Harry M.T. Choi, ${ }^{3}$ Carol Readhead ${ }^{2,3}$ Scott E. Fraser, ${ }^{2}$ Niles A. Pierce, ${ }^{3,4}$ and Rusty Lansford ${ }^{1,5}$ \\ ${ }^{1}$ Keck School of Medicine, University of Southern California, Children's Hospital Los Angeles, California 90027; \\ ${ }^{2}$ Translational Imaging Center, University of Southern California, Los Angeles, California 90089; ${ }^{3}$ Division of \\ Biology \& Biological Engineering, California Institute of Technology, Pasadena, California 91125; ${ }^{4}$ Division of \\ Engineering \& Applied Science, California Institute of Technology, Pasadena, California 91125
}

Multiplexed fluorescent hybridization chain reaction (HCR) and advanced imaging techniques can be used to evaluate combinatorial gene expression patterns in whole mouse embryos with unprecedented spatial resolution. Using HCR, DNA probes complementary to mRNA targets trigger chain reactions in which metastable fluorophore-labeled DNA HCR hairpins self-assemble into tethered fluorescent amplification polymers. Each target mRNA is detected by a probe set containing one or more DNA probes, with each probe carrying two HCR initiators. For multiplexed experiments, probe sets for different target mRNAs carry orthogonal initiators that trigger orthogonal DNA HCR amplification cascades labeled by spectrally distinct fluorophores. As a result, in situ amplification is performed for all targets simultaneously, and the duration of the experiment is independent of the number of target mRNAs. We have used multiplexed fluorescent in situ HCR and advanced imaging technologies to address questions of cell heterogeneity and tissue complexity in craniofacial patterning and anterior neural development. In the sample protocol presented here, we detect three different mRNA targets: $\mathrm{Tg}$ (egfp), encoding the enhanced green fluorescent protein (GFP) transgene (typically used as a control); Twist1, encoding a transcription factor involved in cell lineage determination and differentiation; and Pax2, encoding a transcription factor expressed in the mid-hindbrain region of the mouse embryo.

It is essential that you consult the appropriate Material Safety Data Sheets and your institution's Environmental Health and Safety Office for proper handling of equipment and hazardous materials used in this protocol.

RECIPES: Please see the end of this protocol for recipes indicated by $<R>$. Additional recipes can be found online at http://cshprotocols.cshlp.org/site/recipes.

Reagents

Amplification buffer for HCR $<\mathrm{R}>$

This solution is available from Molecular Instruments (www.molecularinstruments.org).

DNA HCR amplifier sequences ( $3 \mu \mathrm{m}$ stocks in TE containing $150 \mathrm{mM} \mathrm{NaCl}$ )

Each DNA HCR amplifier (Dirks and Pierce 2004) comprises two fluorophore-labeled hairpin molecules (H1 and H2) that undergo conditional polymerization in response to detection of initiators (I1 or I2) carried by the probes. Amplifier sequences are described by Choi et al. (2014). Validated fluorophore-labeled DNA HCR amplifiers were synthesized and purified by Molecular Instruments (Choi et al. 2014).

\footnotetext{
${ }^{5}$ Correspondence: lansford@usc.edu

(c) 2015 Cold Spring Harbor Laboratory Press

Cite this protocol as Cold Spring Harb Protoc; doi:10.1101/pdb.prot083832
} 
D. Huss et al.

DNA probe sequences ( $1 \mu \mathrm{M}$ stocks in TE)

In the sample protocol presented here, each of the three target $m R N A$ s is detected with a different probe set (see Table 1). Each probe set contains multiple DNA probes; each probe carries two HCR initiators (I1 and I2). Within a given probe set, each probe initiates the same HCR amplifier. The DNA probes were designed, synthesized, and purified by Molecular Instruments (Choi et al. 2014).

Methanol (J.T. Baker 9070-01)

Mounting medium (PermaFluor Aqueous Mounting Medium [Fisher Scientific TA-030-FM])

Mouse (pregnant female)

Transgenic mice expressing a control mRNA (e.g., egfp) can be useful when validating in situ HCR.

Nail polish

Paraformaldehyde (PFA) for HCR $(4 \%)<\mathrm{R}>$

PBST for HCR $<$ R $>$

Probe hybridization buffer for $\mathrm{HCR}<\mathrm{R}>$

This solution is available from Molecular Instruments.

Probe wash buffer for HCR $<\mathrm{R}>$

This solution is available from Molecular Instruments.

RNaseZap (Ambion/Life Technologies AM9780)

ScaleA2 $<\mathrm{R}>$

SSCT for HCR $(5 x)<\mathrm{R}>$

Vaseline or silicone grease

$\ldots$ Equipment

Forceps (\#5; Fine Science Tools 11295-10)

Fume hood or histology slot hood (see Step 4)

Glass coverslips $(22 \times 22 \mathrm{~mm}$; No. 1$)$

Glass slides $(25 \times 75 \mathrm{~mm})$

Glass vials (1.5-dram)

Heat block

HybAid oven

Ice bucket

Imaging equipment

In the sample protocol presented here, all images were acquired using a Zeiss 780 NLO inverted confocal microscope with an LD LCI Plan-Apochromat 25x/0.8 DIC Imm Corr (UV) VIS-IR. The ZEN software was used to control microscope components, and the Zeiss MultiTime macro was used to gather tiles of stacks of optical sections.

Microcentrifuge tubes (1.5-mL; RNase-free)

Petri dishes (10-cm; RNase-free)

Transfer pipettes

\section{In Situ HCR}

The following in situ HCR protocol is adapted from Choi et al. (2014) for use in whole-mount mouse embryos. The overall scheme for multiplexed fluorescent HCR (Steps 9-20) is summarized in Figure 1.

Mapping the expression of a transgenic target $m R N A$ provides a useful positive and negative control when validating in situ HCR in a new organism. Figure 2 shows staining for $\mathrm{Tg}(\mathrm{egfp})$ driven by Wnt1 regulatory machinery for wild-type (Target - ) and transgenic (Target +) mouse embryos.

\section{Embryo Preparation}

1. Wipe all dissection equipment with RNaseZap. 


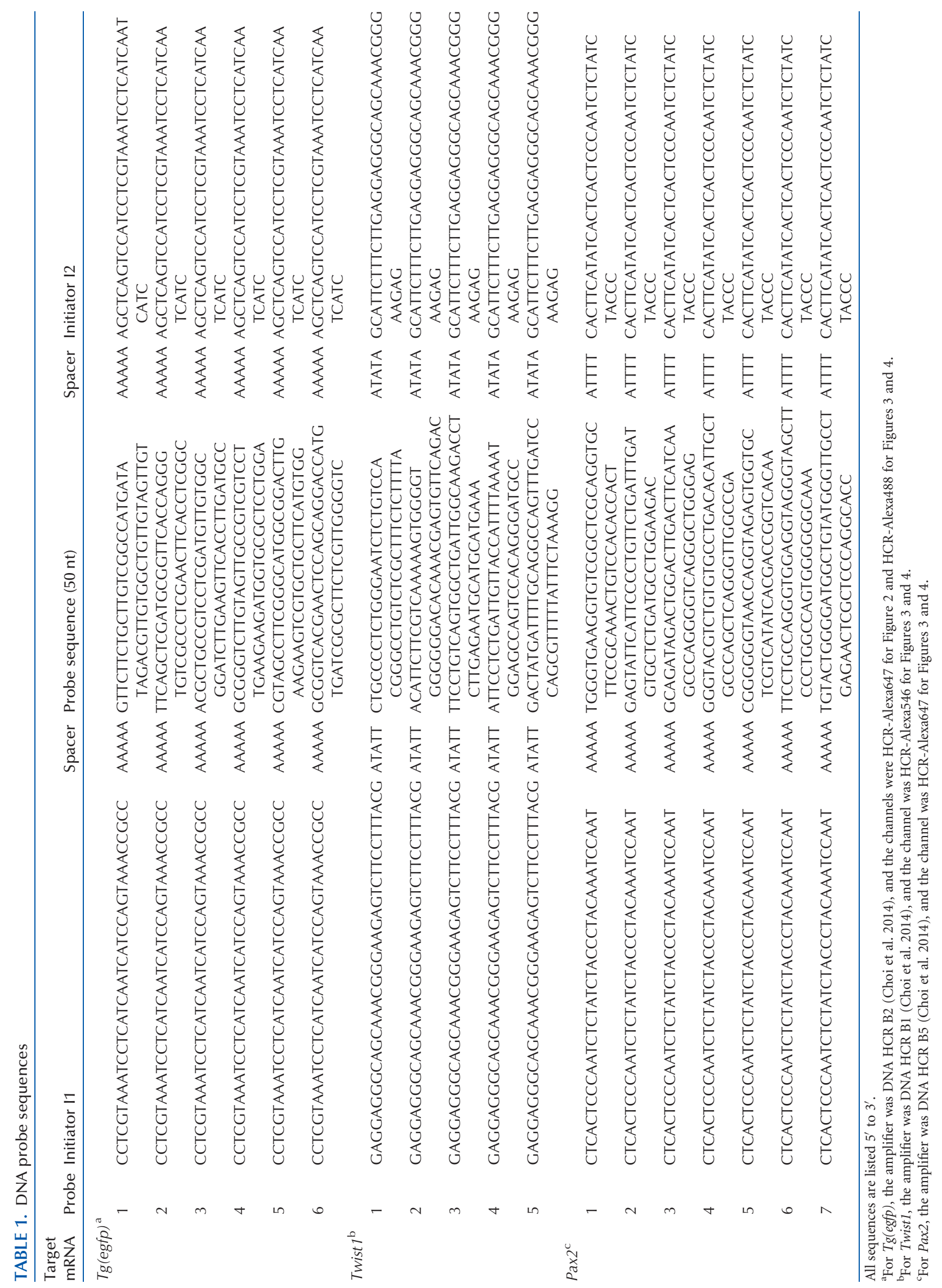


A

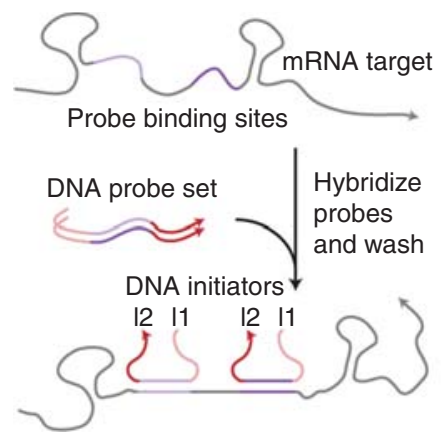

B

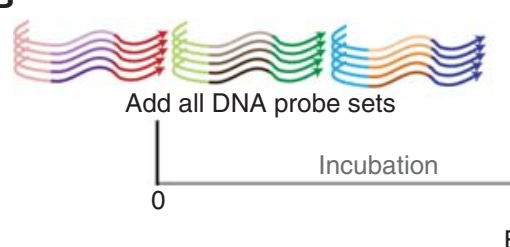

Amplification stage
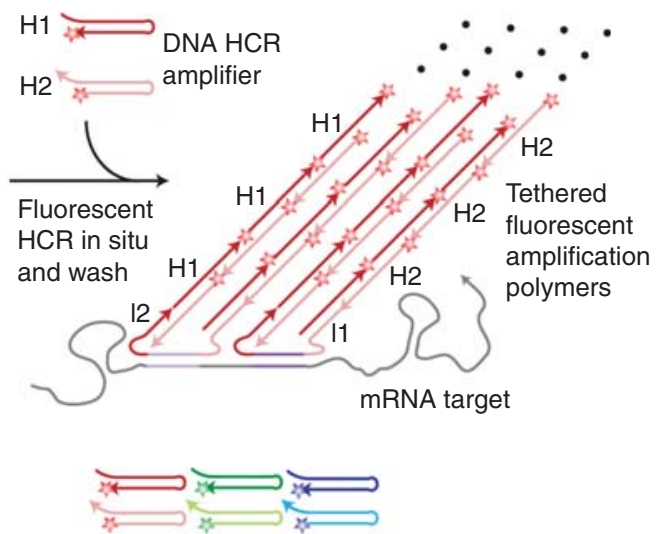

Add all DNA HCR amplifiers

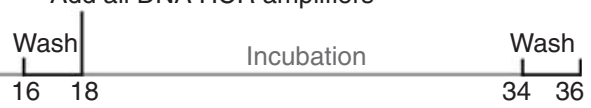

Experimental time (h)

FIGURE 1. Fluorescent in situ HCR. (A) Protocol summary. In the detection stage, probe sets are hybridized to mRNA targets, and unused probes are washed from the sample. In the amplification stage, initiators trigger self-assembly of tethered fluorescent amplification polymers, and unused hairpins are washed from the sample. $(B)$ Experimental timeline. The same two-stage protocol is used independent of the number of target mRNAs. For multiplexed experiments (here, a three-color example is depicted), probe sets for different target mRNAs (five probes depicted per set) carry orthogonal initiators that trigger orthogonal HCR amplification cascades labeled by spectrally distinct fluorophores. (Image from Choi et al. 2014; http://pubs.acs.org/doi/abs/10.1021/nn405717p; used with permission of the American Chemical Society.)

A

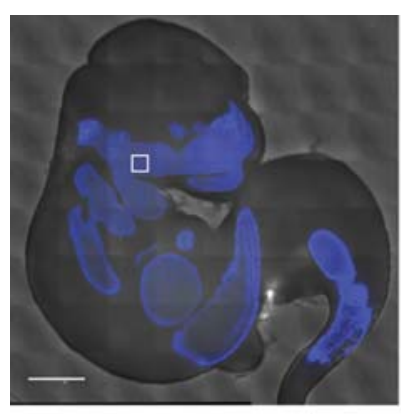

$\underline{\text { Signal + Background }}$

Target +
Replicate 2
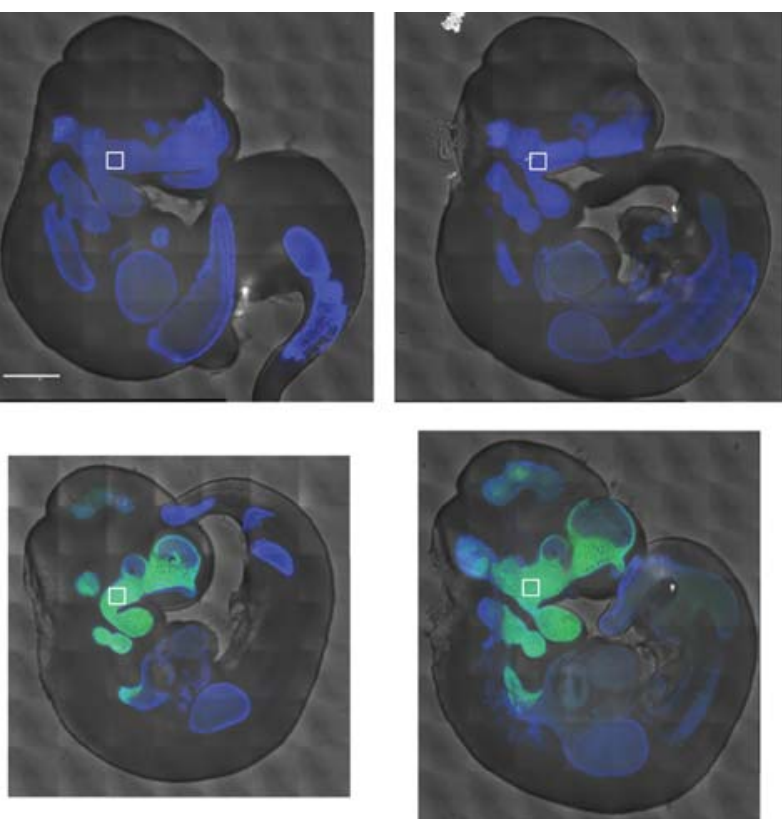
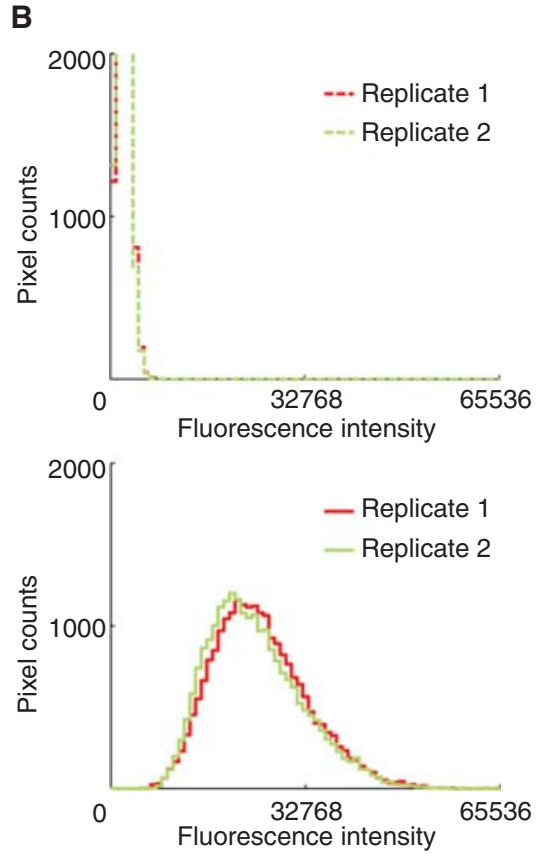

FIGURE 2. Validation of fluorescent in situ HCR by mapping expression of a transgenic target mRNA in wild-type and transgenic whole-mount mouse embryos. (A) mRNA expression is imaged by confocal microscopy. Target: Transgenic mRNA $\operatorname{Tg}($ egfp). Sample: Wild-type embryos lacking the target (Target -) or transgenic embryos containing the target $($ Target + ) fixed at E10.5. For each replicate, the in situ protocol was performed on wild-type and transgenic embryos mixed in the same tube. Green channel: HCR-Alexa647. Blue channel: DAPI. Gray channel: phase. Scale bar, 500 $\mu \mathrm{m}$. $(B)$ Pixel intensity histograms plot the background (in wild-type embryos lacking the target; depicted by white squares) and signal plus background (in transgenic embryos containing the target; depicted by white squares). 
2. Kill a pregnant female mouse using an IACUC-approved protocol.

3. Immediately remove the uterus and submerge it in 4\% PFA in a fresh RNase-free Petri dish.

4. Dissect the mouse embryos from the uterus while it is submerged in 4\% PFA.

This step should be performed with active ventilation from a fume hood or a histology slot hood to protect oneself from exposure to the fixative fumes.

Each female mouse produces $\sim 6-9$ embryos. For Steps 5-9, we recommend using $\sim 1 \mathrm{~mL}$ of solution per group of 10 embryos.

5. Transfer the embryos to a clean vial with fresh $4 \%$ PFA and fix them overnight or longer at $4^{\circ} \mathrm{C}$.

6. Wash the embryos twice in PBST for 10 min each at room temperature.

7. Dehydrate the embryos through the following graded series of methanol/PBST washes. Perform each wash for $10 \mathrm{~min}$ at room temperature. (Incubate embryos older than E10.5 for a longer period in each wash.)

i. $25 \%$ methanol/75\% PBST

ii. 50\% methanol/50\% PBST

iii. $75 \%$ methanol/25\% PBST

iv. $100 \%$ methanol

v. $100 \%$ methanol

8. Store the embryos in $100 \%$ methanol overnight (at least $16 \mathrm{~h}$ ) or until use at $-20^{\circ} \mathrm{C}$.

\section{Detection Stage: Probe Hybridization}

9. Rehydrate the embryos through the following graded series of methanol/PBST washes. Perform each wash for $10 \mathrm{~min}$ at room temperature.

i. $75 \%$ methanol $/ 25 \%$ PBST

ii. 50\% methanol/50\% PBST

iii. $25 \%$ methanol/75\% PBST

iv. $100 \%$ PBST

v. $100 \%$ PBST

10. Immerse the embryos in probe hybridization buffer and prehybridize them for $2 \mathrm{~h}$ at $65^{\circ} \mathrm{C}$. A volume of $500 \mu \mathrm{L}$ of hybridization buffer in a 1.5-dram glass vial is sufficient to cover the embryos.

11. Prepare the probe solution by adding 1 pmol of each probe ( $1 \mu \mathrm{L}$ of each $1 \mu \mathrm{M}$ stock) to $500 \mu \mathrm{L}$ of probe hybridization buffer at $45^{\circ} \mathrm{C}$.

12. Remove the prehybridization buffer from the embryos and add the probe solution.

13. Incubate the embryos overnight (for $12-16 \mathrm{~h}$ ) at $45^{\circ} \mathrm{C}$.

14. Remove the excess probes with the following sequence of washes. Perform the washes at $45^{\circ} \mathrm{C}$. Use $1 \mathrm{~mL}$ of probe wash buffer for each wash, changing the buffer each time.

i. $2 \times 5 \mathrm{~min}$

ii. $2 \times 30 \mathrm{~min}$

iii. $1 \times 5$ min

Longer wash times can be used if necessary.

Proceed immediately to Step 15.

\section{Amplification Stage: HCR Polymerization}

15. Preamplify the embryos in $500 \mu \mathrm{L}$ of amplification buffer for $30 \mathrm{~min}$ at room temperature. 
D. Huss et al.

16. Prepare $30 \mathrm{pmol}$ of each fluorescently labeled hairpin by heating $10 \mu \mathrm{L}$ of each $3 \mu \mathrm{m}$ amplifier stock to $95^{\circ} \mathrm{C}$ for $90 \mathrm{sec}$ and then allowing the samples to cool to room temperature in the dark for $30 \mathrm{~min}$.

Ensure the proper amplifier system (in this example protocol, B1, B2, and B5 [see Table 1]) is used for each probe set and that the amplifiers have Alexa Fluor dyes that are spectrally distinct for each target $m R N A$ in a multiplexed experiment. Keeping the samples in the dark minimizes photobleaching.

17. Prepare the hairpin solution by adding all cooled hairpin samples to $500 \mu \mathrm{L}$ of amplification buffer at room temperature.

18. Remove the preamplification buffer from the embryos and add the hairpin solution.

19. Incubate the embryos in the dark overnight (12-16 h) at room temperature.

20. Remove excess hairpins with the following sequence of washes. Perform the washes with agitation and/or rocking in the dark at room temperature. Use $1 \mathrm{~mL}$ of $5 \times$ SSCT for each wash, changing the solution each time.
i. $2 \times 5 \mathrm{~min}$
ii. $2 \times 30 \mathrm{~min}$
iii. $1 \times 5 \mathrm{~min}$

Longer wash times can be used if necessary.
The embryos can be stored for at least $1 \mathrm{wk}$ at $4^{\circ} \mathrm{C}$ in the dark.

Clearing and Mounting Embryos

21. Clear the embryos for imaging by incubating them in $500 \mu \mathrm{L}$ of ScaleA2 for $2 \mathrm{~d}$ in the dark at room temperature.

22. Clean all glass slides and coverslips with RNaseZap. Add four beads of vaseline to each slide to support a coverslip at the corners.

23. Transfer the embryos onto a slide. Orient the embryos and remove excess buffer.

In general, two embryos can be mounted onto each slide.

24. Add two drops of fluorescent mounting medium over the embryos on each slide.

Alternatively, embryos can be mounted in ScaleA2. ScaleA2 has refractive indices of 1.382, 1.387, and 1.380 at 589, 486, and $656 \mathrm{~nm}$, respectively (Hama et al. 2011).

25. Place a coverslip over the embryos. Apply enough pressure to push the coverslip onto the embryos without flattening them. Seal the edges of the coverslip with nail polish.

If imaging GFP, apply a minimal amount of nail polish, as excess nail polish will destroy GFP fluorescence.

26. Store the slides in the dark at $4^{\circ} \mathrm{C}$ until imaging.

Image Acquisition

27. Acquire images using an inverted confocal microscope system.

The images in Figures 2-4 were acquired using a Zeiss 780 NLO inverted confocal microscope with an $L D$ LCI Plan-Apochromat 25×/0.8 DIC Imm Corr (UV) VIS-IR (see Table 2 for the excitation lasers and emission filter settings). The ZEN software was used to control microscope components, and the Zeiss MultiTime macro was used to gather tiles of stacks of optical sections.

See Troubleshooting.

Problem (Step 27): The fluorescent signal is not sufficiently strong relative to background fluorescence.

Solution: When mapping the expression pattern for a new target mRNA, we balance brightness, robustness, and cost considerations by using a probe set containing five two-initiator DNA probes (depicted in Fig. 1B). For each of the three target mRNAs in this example protocol, the 
Downloaded from http://cshprotocols.cshlp.org/ on April 26, 2023 - Published by

A
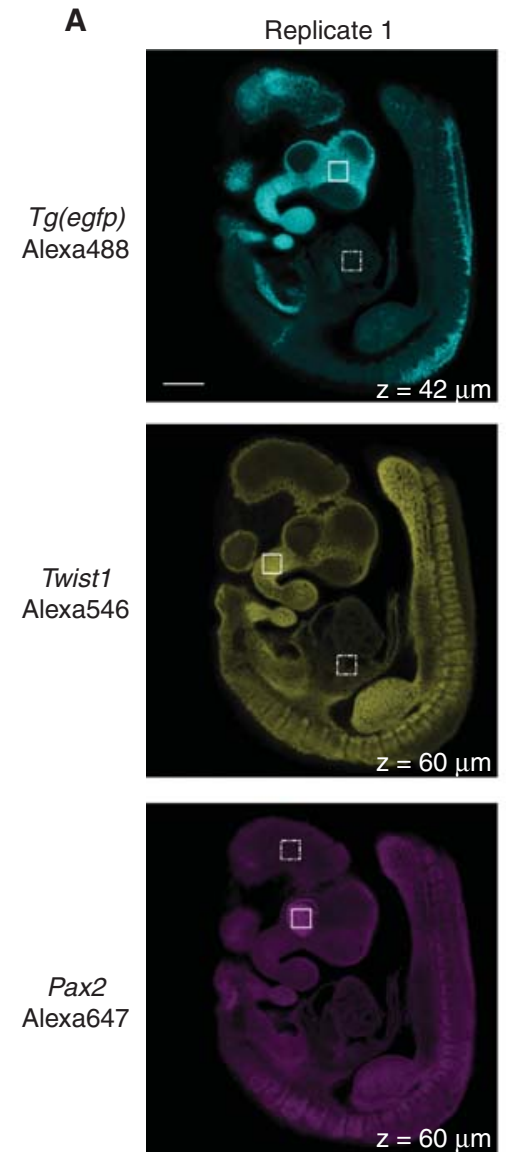
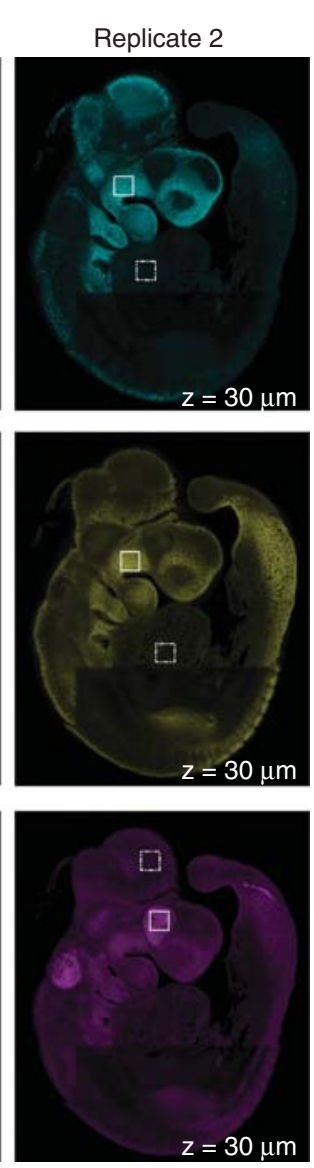

B
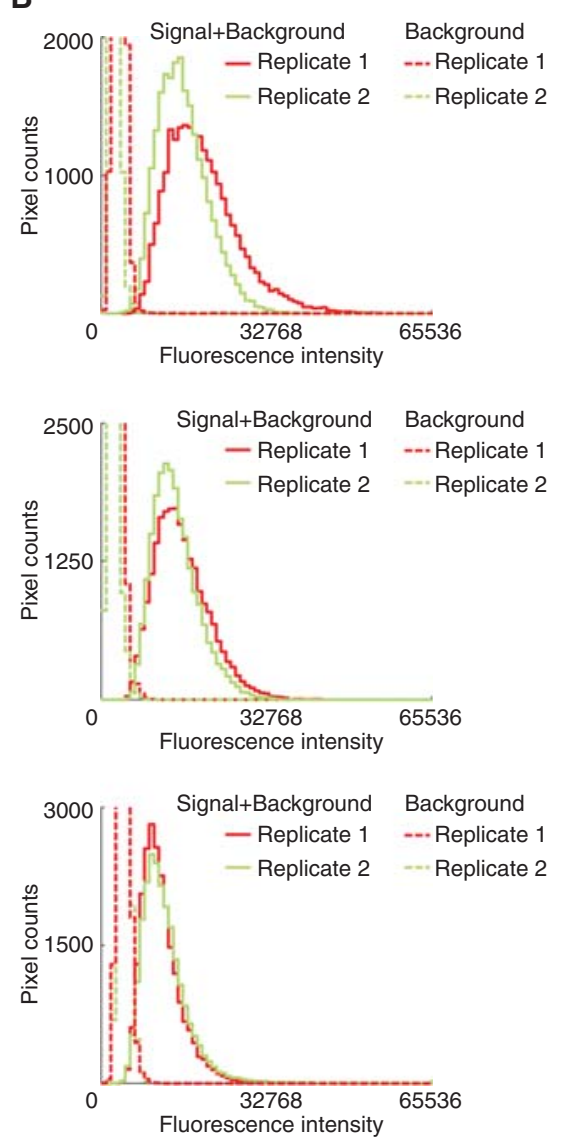

FIGURE 3. Comparison of total fluorescence (signal + background) and background fluorescence pixel intensity distributions for three target mRNAs in whole-mount mouse embryos. (A) Confocal images are shown for three target mRNAs collected in three spectrally distinct fluorescent channels. For each of two embryos, a representative optical section is selected for each channel based on the expression depth of the corresponding target mRNA. Targets: Tg(egfp), Twist1, Pax2. Sample: transgenic whole-mount embryos fixed at E10.5. Cyan channel: HCR-Alexa488 targeting $\mathrm{Tg}$ (egfp). Yellow channel: HCR-Alexa546 targeting Twist1. Magenta channel: HCR-Alexa647 targeting Pax2. Scale bar, $300 \mu \mathrm{m}$. (B) Pixel intensity histograms compare the pixels in two representative squares per image per channel (signal + background [solid line], background [dashed line]).

pixel intensity histograms in Figure 3 show that there is minimal overlap between the distribution of pixel intensities for total fluorescence (signal + background) and the distribution of background fluorescence. If the signal is not sufficiently bright relative to background, we increase the number of probes in the probe set.

Problem (Step 27): Background fluorescence is dominated by nonspecific detection.

Solution: Background may arise from any of three sources: autofluorescence (inherent fluorescence of the fixed sample), nonspecific detection (probes that bind nonspecifically and are subsequently amplified), and nonspecific amplification (HCR hairpins that bind nonspecifically within the sample). If the background is dominated by staining that results from nonspecific detection, we test probes individually to eliminate any that show poor selectivity for the target mRNA.

HCR permits the combinatorial expression patterns of even (low-copy) transcription factors to be detected with high resolution within fixed whole-mount mouse embryos. Figure 4 shows the simul- 
D. Huss et al.
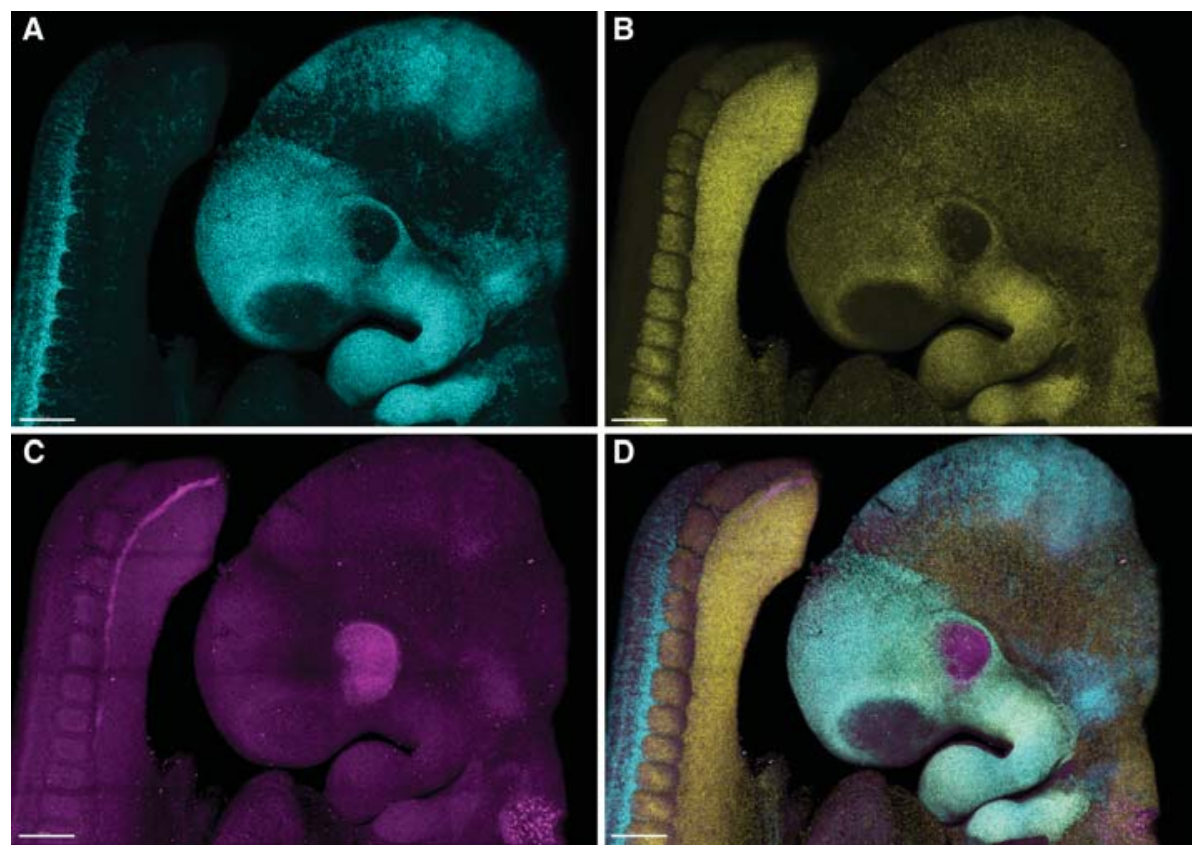

FIGURE 4. Multiplexed high-resolution mapping of complex interlaced and overlapping mRNA expression patterns in the developing mouse head. mRNA expression is imaged by confocal microscopy. Sample: transgenic whole-mount embryos fixed at E10.5. Amplifiers: three orthogonal DNA HCR amplifiers carrying spectrally distinct fluorophores. $(A)$ Cyan channel: HCR-Alexa488 targeting $\operatorname{Tg}($ egfp). (B) Yellow channel: HCR-Alexa546 targeting Twist1. (C) Magenta channel: HCR-Alexa647 targeting Pax2. (D) Composite image. Scale bar, $200 \mu \mathrm{m}$.

taneous mapping of three target mRNAs in the head of a whole-mount mouse embryo, revealing complex interlaced and overlapping expression patterns with high resolution. This HCR protocol improves on the original RNA HCR technology (Choi et al. 2010), using next-generation DNA probes and DNA HCR amplifiers to achieve higher gain, lower cost, and greater durability (Choi et al. 2014). Because amplification is performed in parallel for all targets, the approach is faster and more versatile than traditional in situ hybridization (ISH) approaches that require serial mapping of one target after another. With HCR, the expression patterns of up to five mRNAs can be mapped simultaneously.

One of the challenges of studying neural development is its sheer complexity, due in large part to the number and diversity of its various components at scales ranging from molecular to cellular to tissue. Craniofacial and anterior neural development results from the patterning of the skull and the brain it houses, which in turn directs other craniofacial structures to communicate, chew, taste, hear, balance, and smell the surrounding environment. Regionalization and cell fate specification are critical events within developing neural regions that require precise spatiotemporal regulation of gene expression patterns and the resulting intercellular and intracellular signals that drive patterning, growth, morphogenesis, and the final fates of brain, vascular, and neural crest cells. While many of the components involved in the multidimensional challenge of craniofacial development have been defined, the full range of the cellular diversity and the roles of the interactions between cells have

TABLE 2. Excitation lasers and emission filter settings used for Figures 2-4

\begin{tabular}{llcc}
\hline Figure no. & Fluorophore & $\begin{array}{c}\text { Excitation laser } \\
\text { source }(\mathrm{nm})\end{array}$ & $\begin{array}{c}\text { Tuned emission } \\
\text { bandpass filter }(\mathrm{nm})\end{array}$ \\
\hline Figure 2 & DAPI & 405 & $425-516$ \\
Figures 3 and 4 & Alexa647 & 633 & $640-721$ \\
& Alexa488 & 488 & $494-554$ \\
& Alexa546 & 561 & $562-660$ \\
& Alexa647 & 633 & $653-686$ \\
\hline
\end{tabular}


only begun to be illuminated. Single-cell approaches, in which the coexpression of key components in the same cells can be defined, are required for this field to advance. Using state-of-the-art HCR and molecular imaging approaches, it will be possible to systematically image and genetically probe neural development in mouse embryos to determine the function of downstream molecular pathways essential for proper neural development.

\section{RECIPES}

\section{Amplification Buffer for HCR}

$5 \times$ SSC (Invitrogen/Life Technologies 15557-044)

0.1\% Tween 20 (Bio-Rad 170-6531)

$10 \%$ dextran sulfate (Sigma-Aldrich D6001)

Paraformaldehyde (PFA) for HCR (4\%)

4\% paraformaldehyde (PFA)

$1 \times$ phosphate-buffered saline (PBS) for HCR (DEPC-treated; pH 7.4)

In a fume hood, weigh $1.6 \mathrm{~g}$ of crystalline PFA (Sigma-Aldrich P6148) into a 50-mL conical tube. Add $1 \times$ DEPC-treated PBS ( $\mathrm{pH} 7.4$ ) until the final volume is $40 \mathrm{~mL}$. Heat the solution in a water bath $\left(<60^{\circ} \mathrm{C}\right)$ until all of the PFA powder is completely dissolved. Cool the solution on ice and store at $-20^{\circ} \mathrm{C}$ until use. Thaw and use the fixative only once; dispose of the remaining fixative according to local ordinances.

\section{PBST for HCR}

$1 \times$ phosphate-buffered saline (PBS) for HCR (DEPC-treated; pH 7.4)

$1 \%$ Tween 20

Add $10 \mathrm{~mL}$ of Tween 20 (Bio-Rad 170-6531) and $100 \mathrm{~mL}$ of $10 \times$ PBS to $890 \mathrm{~mL}$ of ultrapure distilled $\mathrm{H}_{2} \mathrm{O}$ (Invitrogen 10977-023). Filter the solution through a $0.2-\mu \mathrm{m}$ filter and store it at $4^{\circ} \mathrm{C}$ until use.

Phosphate-Buffered Saline (PBS) for HCR (10×, pH 7.4)

$1.37 \mathrm{M} \mathrm{NaCl}$

$27 \mathrm{~mm} \mathrm{KCl}$

$80 \mathrm{mM} \mathrm{Na}_{2} \mathrm{HPO}_{4}$

$20 \mathrm{mM} \mathrm{KH}_{2} \mathrm{PO}_{4}$

To prepare $1 \mathrm{~L}$ of a $10 \times$ stock solution, combine $80 \mathrm{~g}$ of $\mathrm{NaCl}$ (Sigma-Aldrich S3014), $2 \mathrm{~g}$ of $\mathrm{KCl}$ (Sigma-Aldrich P9541), $11.4 \mathrm{~g}$ of $\mathrm{Na}_{2} \mathrm{HPO}_{4}$ (anhydrous; Sigma-Aldrich S3264), and $2.7 \mathrm{~g}$ of $\mathrm{KH}_{2} \mathrm{PO}_{4}$ (anhydrous; Sigma-Aldrich P9791). Adjust the $\mathrm{pH}$ to 7.4 with $\mathrm{HCl}$, and bring the final volume to $1 \mathrm{~L}$ with ultrapure $\mathrm{H}_{2} \mathrm{O}$. Avoid using $\mathrm{CaCl}_{2}$ and $\mathrm{MgCl}_{2}$ in PBS for HCR. It is important that the PBS for HCR is prepared as an RNase-free solution (e.g., via diethylpyrocarbonate [DEPC] treatment).

\section{Probe Hybridization Buffer for HCR}

50\% formamide, deionized (Ambion AM9342)

$5 \times$ SSC (Invitrogen/Life Technologies 15557-044)

$9 \mathrm{~mm}$ citric acid (pH 6.0) (Sigma-Aldrich C2404)

0.1\% Tween 20 (Bio-Rad 170-6531)

$50 \mu \mathrm{g} / \mathrm{mL}$ heparin (Sigma-Aldrich H3393)

$1 \times$ Denhardt's solution (Invitrogen/Life Technologies 750018)

10\% dextran sulfate (Sigma-Aldrich D6001) 
D. Huss et al.

Probe Wash Buffer for HCR

$50 \%$ formamide, deionized (Ambion AM9342)

$5 \times$ SSC (Invitrogen/Life Technologies 15557-044)

$9 \mathrm{~mm}$ citric acid ( $\mathrm{pH}$ 6.0) (Sigma-Aldrich C2404)

0.1\% Tween 20 (Bio-Rad 170-6531)

$50 \mu \mathrm{g} / \mathrm{mL}$ heparin (Sigma-Aldrich H3393)

ScaleA2

4 м urea

$10 \%$ glycerol

0.1\% Triton X-100 (Sigma-Aldrich T8787)

ScaleA2 has a $\mathrm{pH}$ of 7.7 .

SSCT for HCR (5×)

$5 \times$ SSC (Invitrogen/Life Technologies 15557-044)

0.1\% Tween 20 (Bio-Rad 170-6531)

\section{ACKNOWLEDGMENTS}

This work draws on molecular architectures developed within the NSF Molecular Programming Project (NSF-CCF-0832824 and NSF-CCF-1317694) and was funded by the NIDCR FaceBase Consortium (U01-DE020063), the NIH (5R01EB006192), the Gordon and Betty Moore Foundation (GBMF2809), the Beckman Institute at Caltech (Programmable Molecular Technology Center).

\section{REFERENCES}

Dirks RM, Pierce NA. 2004. Triggered amplification by hybridization chain reaction. Proc Natl Acad Sci 101: 15275-15278.

Choi HMT, Chang JY, Trinh LA, Padilla JE, Fraser SE, Pierce NA. 2010. Programmable in situ amplification for multiplexed imaging of mRNA expression. Nat Biotechnol 28: 1208-1212.

Choi HMT, Beck VA, Pierce NA. 2014. Next-generation HCR in situ hybridization chain reaction: Higher gain, lower cost, greater durability. ACS Nano 8: 4284-4294.
Hama H, Kurokawa H, Kawano H, Ando R, Shimogori T, Noda H, Fukami K, Sakaue-Sawano A, Miyawaki A. 2011. Scale: A chemical approach for fluorescence imaging and reconstruction of transparent mouse brain. Nat Neurosci 14: 1481-1488. 


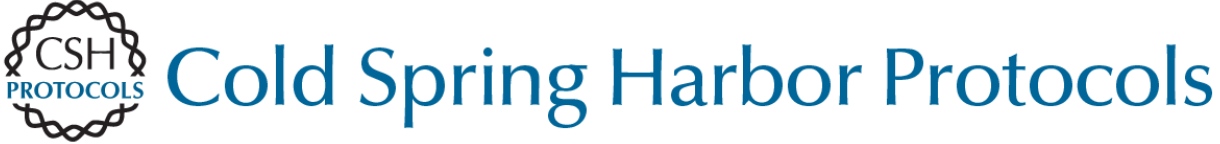

\section{Combinatorial Analysis of mRNA Expression Patterns in Mouse Embryos Using Hybridization Chain Reaction}

David Huss, Harry M.T. Choi, Carol Readhead, Scott E. Fraser, Niles A. Pierce and Rusty Lansford

Cold Spring Harb Protoc; doi: 10.1101/pdb.prot083832

\begin{tabular}{cc}
$\begin{array}{c}\text { Email Alerting } \\
\text { Service }\end{array}$ & Receive free email alerts when new articles cite this article - click here. \\
\hline $\begin{array}{c}\text { Subject } \\
\text { Categories }\end{array}$ & $\begin{array}{c}\text { Browse articles on similar topics from Cold Spring Harbor Protocols. } \\
\text { Analysis of Gene Expression (197 articles) } \\
\text { Analysis of Gene Expression, general (129 articles) } \\
\text { Confocal Microscopy (114 articles) } \\
\text { Imaging Development (255 articles) } \\
\text { Imaging for Neuroscience (342 articles) } \\
\text { In Situ Hybridization (93 articles) } \\
\text { Mouse (437 articles) Expression (127 articles) } \\
\text { Visualization of Gene Expression }\end{array}$ \\
\hline
\end{tabular}

